\title{
Teaching research of computer operating system based on analogy teaching method
}

\author{
Qianqian Luan, Jiuru Wang*, Lihua Han, Zhenhai Wang \\ ${ }^{1}$ School of information science and engineering, linyi university, linyi 276000, China \\ \{luanqianqian, wangjiuru, hanlihua, wangzhenhai\} @ lyu.edu.cn \\ ${ }^{*}$ Corresponding author
}

Keywords: computer operating system; analogies; the curriculum reform

\begin{abstract}
The design mode of computer operating system fully conforms to the thinking characteristics of human society. Computer operating system is not only a basic course of computer science and technology or other related majors, but also the best course for students to understand the thinking mode of computer and train innovation sense. However, computer operating system contains many concepts and theories, students understand a little difficult. Based on the teaching experience of research group for many years, it is found that it is easy for students to understand and master the knowledge points by using vivid examples in life and creating teaching situations to simulate typical design ideas and implementation methods. The research group put forward the training system of computer operating system with "one center and three basic points". It is not only beneficial to cultivating students' innovative and entrepreneurial thinking, but also enlightening to students' understanding of daily life problems.
\end{abstract}

\section{基于类比教学法的《计算机操作系统》课程教学研究}

\author{
奕倩倩, 王九如*, 韩丽花, 王振海 \\ 临沂大学 信息科学与工程学院, 山东 临沂 276000 \\ \{luanqianqian, wangjiuru, hanlihua, wangzhenhai\}@lyu.edu.cn
}

关键字：计算机操作系统；类比教学法；课程改革

摘要：计算机操作系统设计模式完全符合人类社会的思维特征，《计算机操作系统》课程既 是计算机科学与技术及相关专业的专业基础课, 又是学生理解计算机思维模式、培养创新意 识的最佳课程。但是《计算机操作系统》本身概念多、理论性强, 学生理解有些吃力。依据 课题组多年一线教学经验, 发现利用生活中生动的实例类比操作系统中典型的设计思路与实 现方法, 创设教学情境, 易于学生理解和掌握知识点。课题组提出 “一个中心, 三个基本点” 的《计算机操作系统》培养体系, 既对培养学生创新创业思维有益、又对学生通过学习《计 算机操作系统》领悟日常生活问题处理有启迪。

\section{1.引言}

《计算机操作系统》是计算机科学与技术及相关专业的专业基础课，它管理着计算机系 统的软硬件资源。ACM和IEEE/CS 提交的计算学科本科教学指导 《Computinger Science Curricula 2013》, 明确指出“计算机操作系统是最基础和最重要的课程之一”。《计算机操作 系统》把计算机理论与实践融为一体, 在计算机科学与技术中起着不可替代的承前启后作 用。由于计算机操作系统设计模式完全符合人类社会的思维特征, 因此深入学习《计算机操 
作系统》不仅对学生了解计算机体系结构、从事计算机系统编程有着非常重要的作用; 而且 对培养学生发现问题的能力, 培育创新思维模式有着很好的帮助, 是计算机思维模式、创新 教育最好的体现。

但是，在教学实践活动中发现：学生普遍反映本课程概念多、知识点多、内容比较抽象、 理解比较吃力。究其原因主要有三个方面: (1)从教材本身来讲, 《计算机操作系统》关联多 门课程, 有较强的理论性和综合性, 要求学生有较强的抽象思维能力、程序设计能力、及相 应的硬件基础知识; (2)从学生角度来讲, 个别学生认为该课程没有用, 不像 $\mathrm{C}$ 语言等课程有 立竿见影的效果; (3)从教师角度来讲, 课程学术性强, 传统教学过程中受课时所限难以充分 论述。

为克服上述问题，根据课题组多年一线教学经验发现：利用生活中生动的例子类比操作 系统中经典设计, 学生更易于理解和掌握知识点。类比教学法是以“建构主义”理论为理论支 撑, 通过把教学内容类比于学生较熟悉的相似或相通事物, 化抽象为具体、建立知识模型, 以帮助学生实现新知识的深化理解, 促进学生自主学习、培养其创新思维为目标的教学方法。 本文按照工程教育认证要求，探索建立一套具有专业特色、风格鲜明的《计算机操作系统》 课程教学体系。该体系概括为“一个中心，三个基本点”，即：“以研究《计算机操作系统》类 比教学法为中心, 组织一套系统精炼的教学内容, 设计一套科学可行的考核办法, 塑造一支 结构合理的教师队伍”。把培养学生操作系统应用能力放在重要位置, 研究成果将改变传统操 作系统教学模式和教学内容, 调动学生主动学习的积极性, 为建立以应用型人才培养为目标 的实用教材体系提供参考。

\section{2.《计算机操作系统》教学特点}

\section{1 课程特点}

《计算机操作系统》国内外高校本科教材基本是围绕：处理机管理、存储器管理、设备 管理、文件管理和操作系统的接口五大模块展开。主要在实验平台建设方面, 存在差异。国 外高校更重视从实践中学习理论(Learning By Doing), 通常要求学生做一个简易操作系统。国 内高校普遍以 Linux、或 Windows 为实验平台的开展验证性实验。

《计算机操作系统》课程与其前驱课程关系密切。《计算机程序设计语言基础》、《计 算机组成原理》、《数据结构》、《电子技术》、以及《高等数学》等都《计算机操作系统》 的前驱课程, 这些基础课程的掌握程度, 对掌握《计算机操作系统》知识有着非常重要的影 响。而且, 在有限的学时内学生既要学习处理机管理、存储器管理、I/O 设备管理、文件管理 等内容，又要理解每一种设计理念的发展历程，对逻辑思维能力要求高。

\section{2 学生特点}

随着计算机专业的蓬勃发展, 招生规模不断扩大, 学生越来越多。学生层次不同, 知识 接受能力存在差异。以笔者所在临沂大学信息科学与工程学院为例, 每个年级的学生大概有 700 人左右, 生源涵盖本科、专科、专升本三个层次, 在学习《计算机操作系统》过程中知 识接受能力差别比较大。比如专升本的学生, 由于在专科时都已经学习过《计算机操作系统》 课程, 本科阶段再次重新学习该课程时理解能力就强很多。而专科学生, 由于高中时所学计 算机知识很少, 大学阶段理论教学相对于本科略浅, 以致接受知识能力较弱。因此, 由于学 生多，知识层次不齐，所以教师在讲解时特别吃力，而且有时事倍功半。

\section{3 考核方式}

由于该课程理论性强, 比较难学。学生真正感兴趣的并不多, 许多同学抱着“及格就行” 的态度来学习。平时不努力, 考试前临时突击蒙混过关。虽然在实际教学过程中, 设有期中 
考试、也有平时考勤成绩, 但最终的学业成绩依然主要由期末考试成绩来考核该课程学习的 好坏。这在一定程度上造成了学生学习浮躁, 不注重平时学习的过程。

\section{3.《计算机操作系统》类别教学法实施方案}

\section{1 教学模块和典型教学案例}

以经典教材西安电子科技大学《计算机操作系统 (第四版) 》为例, 该课程可以划分为 以下五个教学模块, 每个模块都可以借用类比教学案例, 形象而生动的阐述晦涩的科学原理。

第一模块为操作系统引论。主要介绍操作系统的目标、作用、发展过程、基本特性和主 要功能。本模块可以采用软件工程的思路, 把操作系统类比于一个“黑盒”。为了使操作系统 符合方便性、有效性、可扩充性、开放性，以及不断提出的应用需求，从需求分析的角度阐 述“操作系统作为配置在计算机硬件上的第一层软件, 是对硬件系统的首次扩充”, 从设计者 的角度分析操作系统该具有和满足的特性。

第二模块为处理机管理模块, 主要包括进程的描述与控制、处理机调度与死锁。本模块 最为核心的两个内容分别是进程同步和死锁。进程同步既是本模块的重点, 也是《计算机操 作系统》的难点。而进程同步核心问题是分析进程间的关系。为了形象的说明进程间的互斥 关系和同步关系, 可以采用类比教学法以化繁为简。进程互斥关系可以类比为一列火车车厢 中乘客共享卫生间问题。来自五湖四海的乘客，彼此间没有任何联系也互补熟悉，相当于彼 此独立的进程。但由于共享同一个卫生间而产生联系，彼此互斥的使用卫生间。进程同步关 系可以类比于工业流水线。流水线上工人同时处于工作状态，彼此为了完成一项工作而相互 配合，如果前边工人没做完，后续工人就必须等待。在进程同步分析时，可以将数据库“实体 一联系”方法引入计算机操作系统进程同步教学中, 通过将原 E-R 图定义改造为矩形表述进程 实体、菱形表示实体间联系、椭圆表示发生联系的信号量, 可以清晰地表示进程实体间, 以 及进程实体内的联系。

死锁问题可以类比于港台警匪片中坏人因争夺地盘发生僵持——“占有一部分资源，想要 更多资源”，必须有警察出手才能维持秩序化解危机——“借助外力作用”解除死锁。而银行家 算法则可以通过供应链金融加以阐述，使学生理解因资源分配不当造成严重后果。

第三模块为存储管理模块, 包括存储器管理和虚拟存储器两部分, 主要介绍内存连续分 配存储管理方式、分页和分段存储管理方式，以及请求分页和请求分段管理方式。存储管理 模块可以类比于笔记本。宏观的上看, 存储器管理介绍了程序中代码或数据从逻辑地址相邻 到离散的分配方式的转变, 每一次存储技术的革新都是针对以后存储技术不足而提出, 本模 块可以很好地激发学习发现问题、分析问题、解决问题的创新性思维能力。虚拟存储器则很 好地体现了利用存储器的空闲空间分区域存放和运行其他的多道程序, 以此来提高内存利用 率的空分复用技术。

第四模块为 $\mathrm{I} / \mathrm{O}$ 设备管理模块, 讲解操作系统 $\mathrm{I} / \mathrm{O}$ 系统的功能、模型和接口、中断机构 和设备控制器、设备驱动程序、与设备无关的 $I / O$ 软件、用户层的 I/O 软件、缓冲区管理、 以及磁盘存储器的性能和调度。由于计算机设备千差万别, 为了提供统一的解决方案, 本模 块从把各种功能需求从系统的角度按照层次化思想设计开发, 可以把 $\mathrm{I} / \mathrm{O}$ 设备管理类比为人 类社会的分层管理模式, 形象的说明每个层面需要处理的问题。

第五模块为文件管理和磁盘存储, 主要介绍文件和文件系统、文件目录、文件共享、文 件保护、以及外存的组织方式、提高磁盘 I/O 速度和可靠性的技术途径。本模块可以类比为 一个图书馆，为了方便用户查询须采用 “按名存取”，为有效地利用存储空间，需要采用合理 的文件分配方式，改善存储空间的利用率。 


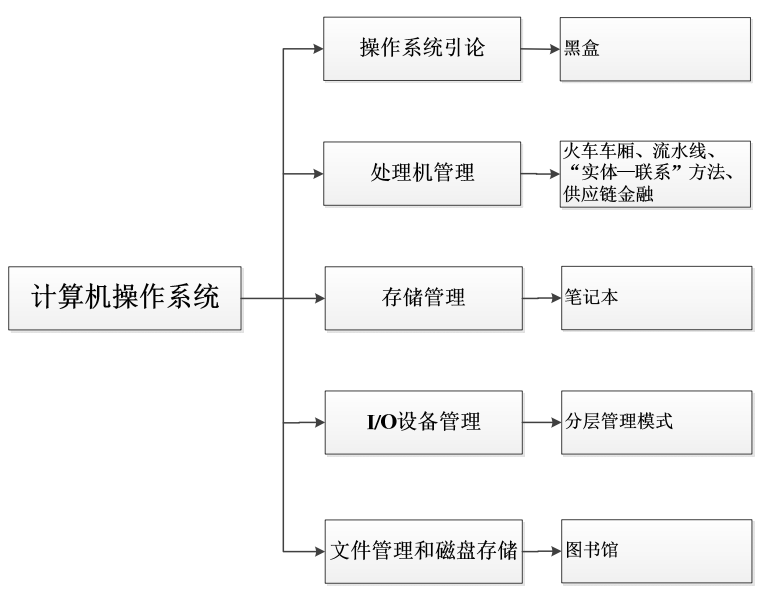

图 1 教学模块与类比案例

\section{2 课程考核方案}

本课程结业考评采用集体命题方式，采用主卷成绩占 70\%，平时成绩占 30\%的方式考核。 主卷：主卷成绩分为课程期末闭卷考试 (50\%) 和实验测试 $(20 \%)$ 二项。平时：平时成绩 分为三部分进行考查，分别为 MOOC 成绩（10\%）、作业成绩（10\%）和实验成绩（10\%）。 MOOC 成绩采用中国大学 MOOC (慕课) 期末成绩, 作业成绩按照历次作业平均值, 实验成 绩采用历次实验报告平均值, 考勤成绩按照上课时老师的点名记录为依据作为督促, 但不记 入学业成绩。

\section{4.小结}

当前, 《计算机操作系统》课程建设与应用型大学人才培养目标存在一定差距问题, 主 要表现在：教学内容、教学方法、考核办法、教师队伍四个方面。因此, 课题组把培养学生 操作系统应用能力放在重要位置, 制定“一个中心, 三个基本点”的研究目标, 借助类比教学 法培养学生学习兴趣和创新创业思维能力, 以改变传统操作系统教学模式和教学内容, 调动 学生主动学习的积极性, 为建立以应用型人才培养为目标的新工科教材体系提供参考。

\section{致谢}

本文为山东省高等教育本科教改项目（M2018X058）的阶段性成果之一。

\section{References}

[1] Zhai Gao-Shi, Xu Hong-Li, Yang Kun, Li De-de. Exploration on the experimental topic of operating system and the construction of teaching ecosystem supporting the research and development of operating system [J]. Industrial and information education,2019(03):44-50.

[2] Wang Qiufen, Wang Yongxin. Reform and practice of teaching method of operating system principle course based on OBE [J]. Education and teaching BBS,2019(12):167-168.

[3] Wei Shaohua. Discussion on teaching method reform of computer operating system [J]. Computer products and circulation,2019(03):239+241.

[4] Tao Yuting, Fang Xuming, Yang Lechan, Liang Yinghong. Suggestions on operating system curriculum reform in local colleges and universities under the background of new engineering [J]. Curriculum education research,2019(09):240-241. 